OPEN ACCESS

Edited by:

Manuel Asorey,

University of Zaragoza, Spain

Reviewed by:

Sania Qureshi,

Mehran University of Engineering and

Technology, Pakistan

Kazuharu Bamba,

Fukushima University, Japan

*Correspondence: Jundong Zhou

zhou109@mail.ustc.edu.cn

Specialty section:

This article was submitted to Mathematical and Statistical Physics,

a section of the journal

Frontiers in Physics

Received: 10 June 2020

Accepted: 17 August 2020

Published: 26 October 2020

Citation:

Zhou J (2020) Rigidity of Complete Minimal Submanifolds in Spheres.

Front. Phys. 8:571250

doi: 10.3389/fphy.2020.571250

\section{Rigidity of Complete Minimal Submanifolds in Spheres}

\author{
Jundong Zhou ${ }^{1,2 *}$ \\ ${ }^{1}$ School of Mathematical Sciences, University of Science and Technology of China, Hefei, China, ${ }^{2}$ School of Mathematics \\ and Statistics, Fuyang Normal University, Fuyang, China
}

Let $M$ be an $n$-dimensional complete minimal submanifold in an $(n+p)$-dimensional sphere $\mathbb{S}^{n+p}$, and let $h$ be the second fundamental form of $M$. In this paper, it is shown that $M$ is totally geodesic if the $L^{2}$ norm of $|h|$ on any geodesic ball of $M$ is of less than quadratic growth and the $L^{n}$ norm of $|h|$ on $M$ is less than a fixed constant. Further, under only the latter condition, we prove that $M$ is totally geodesic. Moreover, we provide a sufficient condition for a complete stable minimal hypersurface to be totally geodesic.

Keywords: rigidity, minimal submanifold, totally geodesic submanifold, stable hypersurface, Sobolev inequality

\section{INTRODUCTION}

Let $(x, u(x))$ be a minimal graph in $\mathbb{R}^{2} \times \mathbb{R}$, which means that $u(x)$ solves the equation

$$
\operatorname{div}\left(\frac{\nabla u}{\sqrt{1+|\nabla u|^{2}}}\right)=0 .
$$

The celebrated Bernstein theorem states that the complete minimal graphs in $\mathbb{R}^{3}$ are planes. The works of Fleming [9], Almgren [1], and Neto and Wang [16] tell us that the Bernstein theorem is valid for complete minimal graphs in $\mathbb{R}^{n+1}$ provided that $n \leq 7$. Counterexamples to the theorem for $n \geq 8$ have been found by Bombieri et al. [2] and, later, by Lawson [13]. On the other hand, do Carmo and Peng [6] and Fischer-Colbrie and Schoen [10] proved independently that a completely stable minimal surface in $\mathbb{R}^{3}$ must be a plane, a result that generalizes the Bernstein theorem. For the high-dimensional case, it is an open question whether the completely oriented stable minimal hypersurfaces in $\mathbb{R}^{n+1}$ (for $3 \leq n \leq 7$ ) are hyperplanes. However, it has been proved by do Carmo and Peng [6] that a complete stable minimal hypersurface $M$ in $\mathbb{R}^{n+1}$ is a hyperplane if

$$
\lim _{R \rightarrow \infty} \frac{\int_{B_{x_{0}}(R)}|h|^{2} d v}{R^{2 q+2}}=0, \quad q<\sqrt{\frac{2}{n}},
$$

where $B_{x_{0}}(R)$ denotes the geodesic ball of radius $R$ centered at $x_{0} \in M$. Many interesting generalizations of the do Carmo-Peng theorem have been obtained (see, e.g., [7, 15, 16, 18]). By definition, the hyperbolic space $\mathbb{H}^{n+p}$ is a Riemannian manifold with sectional curvature -1 which 
is simply connected, complete, and $(n+p)$-dimensional. In hyperbolic space, some results similar to the do Carmo-Peng theorem have been derived. Xia and Wang [20] studied complete minimal submanifolds in a hyperbolic space and obtained the following result.

Theorem 1.1. [20] For $n \geq 5$, let $M$ be an $n$-dimensional complete immersed minimal submanifold in a hyperbolic space $\mathbb{H}^{n+p}$, and let $h$ be the second fundamental form of $M$. Assume that

$$
\lim _{R \rightarrow \infty} \sup \frac{\int_{B_{x_{0}}(R)}|h|^{2} d v}{R^{2}}=0 .
$$

If there exists a positive constant $C$ depending only on $n$ and $p$ such that

$$
\int_{M}|h|^{n} d v<C
$$

then $M$ is totally geodesic.

Recently, de Oliveira and Xia [8] improved Theorem 1.1 as follows.

THEOREM 1.2. [8] For $n \geq 4$, let $M$ be an $n$-dimensional complete immersed minimal submanifold in a hyperbolic space $\mathbb{H}^{n+p}$ such that $n$ and $p$ satisfy $\left(n^{2}-6 n+1\right)+\frac{8}{p}>0$. Assume that

$$
\lim _{R \rightarrow \infty} \sup \frac{\int_{B_{x_{0}}(R)}|h|^{d} d v}{R^{2}}=0,
$$

where $d$ is a constant with the following properties:

(1) if $p=1$ and $n \geq 4$, then

$$
d \in\left(\frac{n-1}{n}, \frac{(n-2)(n-1)}{n}\right) ;
$$

(2) if $p>1$ and $n>5$, then

$$
\begin{aligned}
d \in \frac{(n-1)^{2}}{2 n}(1 & -\sqrt{1-\frac{4}{(n-1)^{2}}\left(n-\frac{2}{p}\right)}, 1 \\
& \left.+\sqrt{1-\frac{4}{(n-1)^{2}}\left(n-\frac{2}{p}\right)}\right) .
\end{aligned}
$$

Then there exists a positive constant $C$ depending only on $n, p$, and $d$ such that $M$ is totally geodesic if

$$
\int_{M}|h|^{n} d v<C
$$

The unit sphere $\mathbb{S}^{n+p}$ is a Riemannian manifold with sectional curvature 1 which is simply connected, complete, and $(n+p)$ dimensional. Many results are available on the classification of compact minimal submanifolds in the unit sphere. Simons [17] calculated the Laplacian of $|h|^{2}$ of minimal submanifolds in a space form. As a consequence of Simons' formula, if $M$ is a compact minimal submanifold in $\mathbb{S}^{n+p}$ and $|h|^{2} \leq \frac{n p}{2 p-1}$, then either $M$ is totally geodesic or $|h|^{2}=\frac{n p}{2 p-1}$. In the latter case, Chern et al. [3] further proved that $M$ is either a Clifford hypersurface or a Veronese surface in $\mathbb{S}^{4}$. Li and $\mathrm{Li}$ [14] and Chen and $\mathrm{Xu}$ [4] proved independently that $M$ is either a totally geodesic submanifold or a Veronese surface in $\mathbb{S}^{4}$ if $|h|^{2} \leq \frac{2}{3} n$ everywhere on $M$. This result improves the pinching constant in Simons' formula. Deshmukh [5] studied $n$-dimensional compact minimal submanifolds in $\mathbb{S}^{n+p}$ with scalar curvature $S$ satisfying the pinching condition $S>n(n-2)$ and proved that for $p \leq 2$ these submanifolds are totally geodesic.

The above results are rigidity theorems valid in the unit sphere, which characterize the behavior of minimal submanifolds. In this paper, we use the methods of minimal submanifolds in Euclidean space and hyperbolic space to investigate the rigidity of complete minimal submanifolds in spherical space. The main theorems are as follows.

THEOREM 1.3. For $n \geq 3$, let $M$ be an $n$-dimensional complete minimal submanifold in the unit sphere $\mathbb{S}^{n+p}$. We further assume that (1.1) holds. If

$$
\int_{M}|h|^{n} d v<C^{n}(n, p)
$$

with $C(n, p)=(c(n))^{-1}\left(n^{2}+7\right)^{-\frac{1}{2}} \sqrt{(2 b(p))^{-1}+(n p b(p))^{-1}}$, where $c(n)=2^{n}(1+n)^{1+\frac{1}{n}}(n-1)^{-1} \omega_{n}^{-\frac{1}{n}}, \omega_{n}$ is the volume of the unit ball in $\mathbb{R}^{n}, b(1)=1$, and $b(p)=\frac{3}{2}$ if $p>1$, then $M$ is totally geodesic.

In [20], Xia and Wang believed that the condition (1.1) is not necessary. It is therefore interesting to see whether we can remove condition (1.1) from Theorem 1.3. In this case, we get a positive answer.

TheOrem 1.4. For $n \geq 3$, let $M$ be an $n$-dimensional complete minimal submanifold in the unit sphere $\mathbb{S}^{n+p}$. If

$$
\int_{M}|h|^{n} d v<\widetilde{C}^{n}(n, p)
$$

with $\widetilde{C}(n, p)=(n c(n))^{-1} \sqrt{\frac{2(n-1)}{\left(n^{2}+7\right) b(p)}+\frac{4}{n\left(n^{2}+7\right) p b(p)}}$, where $c(n)=$ $2^{n}(1+n)^{1+\frac{1}{n}}(n-1)^{-1} \omega_{n}^{-\frac{1}{n}}, \omega_{n}$ is the volume of the unit ball in $\mathbb{R}^{n}, b(1)=1$, and $b(p)=\frac{3}{2}$ if $p>1$, then $M$ is totally geodesic.

Remark 1.5. By using Simons' formula and the technique developed in do Carmo and Peng's paper, we obtain Theorem 1.4. The constant $\widetilde{C}(n, p)$ in Theorem 1.4 is smaller than $C(n, p)$ in Theorem 1.3.

We also investigate stable minimal hypersurfaces in the unit sphere and obtain a result similar to do Carmo and Peng's theorem. A minimal hypersurface $M$ in a Riemannian manifold $N$ is said to be stable if for each $f \in C_{0}^{\infty}(M)$,

$$
\int_{M}\left(|\nabla f|^{2}-\left(|h|^{2}+\overline{\operatorname{Ric}}(v, v)\right) f^{2}\right) d v \geq 0
$$


where $\overline{\text { Ric }}$ is the Ricci curvature of $N$ and $v$ is the unit normal vector of $M$.

THEOREM 1.6. For $n \geq 2$, let $M$ be an $n$-dimensional complete stable minimal hypersurface in the unit sphere $\mathbb{S}^{n+1}$. If

$$
\lim _{R \rightarrow \infty} \sup \frac{\int_{B_{x_{0}}(R)}|h|^{2 \delta} d v}{R^{2}}=0, \quad 1-\sqrt{\frac{2}{n}}<\delta<1+\sqrt{\frac{2}{n}},
$$

where $B_{x_{0}}(R)$ denotes the geodesic ball of radius $R$ centered at $x_{0} \in M$, then $M$ is totally geodesic.

\section{PRELIMINARIES}

Let $M$ be an $n$-dimensional complete submanifold in the $(n+$ $p$ )-dimensional unit sphere $\mathbb{S}^{n+p}$. We will use the following convention on the range of indices unless specified otherwise:

$$
\begin{array}{r}
1 \leq A, B, C, \ldots \leq n+p, \quad 1 \leq i, j, k, \ldots \leq n \\
n+1 \leq \alpha, \beta, \gamma, \ldots \leq n+p .
\end{array}
$$

We choose a local field of orthonormal frame $\left\{e_{1}, e_{2}, \ldots, e_{n+p}\right\}$ in $\mathbb{S}^{n+p}$ such that, restricted to $M,\left\{e_{1}, e_{2}, \ldots, e_{n}\right\}$ is tangent to $M$ and $\left\{e_{n+1}, \ldots, e_{n+p}\right\}$ normal to $M$. Let $\left\{\omega_{A}\right\}$ be the field of dual frame and $\left\{\omega_{A B}\right\}$ the connection 1-form of $\mathbb{S}^{n+p}$. Restricting these forms to $M$, we have

$$
\omega_{i}^{\alpha}=\sum_{j} h_{i j}^{\alpha} \omega_{j}, \quad h=\sum_{i, j, \alpha} h_{i j}^{\alpha} \omega_{i} \otimes \omega_{j} \otimes e_{\alpha}, \quad \xi=\frac{1}{n} \sum_{i, \alpha} h_{i i}^{\alpha} e_{\alpha},
$$

where $h$ and $\xi$ are the second fundamental form and the mean curvature vector of $M$, respectively. We define

$$
H=|\xi|, \quad|h|^{2}=\sum_{i, j, \alpha}\left(h_{i j}^{\alpha}\right)^{2}, \quad|\nabla h|^{2}=\sum_{i, j, k, \alpha}\left(h_{i j k}^{\alpha}\right)^{2}
$$

where $h_{i j k}^{\alpha}$ is the component of the covariant derivative of $h_{i j}^{\alpha}$. When $M$ is minimal, we obtain the Simons' formula $[3,17]$

$$
\begin{aligned}
\frac{1}{2} \Delta|h|^{2} & =|\nabla h|^{2}+n|h|^{2}-\sum_{i, j, k, l, \alpha, \beta} h_{i j}^{\alpha} h_{i j}^{\beta} h_{k l}^{\alpha} h_{k l}^{\beta} \\
& -\sum_{i, j, \alpha, \beta}\left(\sum_{k}\left(h_{i k}^{\alpha} h_{k j}^{\beta}-h_{j k}^{\alpha} h_{k i}^{\beta}\right)\right)^{2} .
\end{aligned}
$$

The last terms in (2.1) can be estimated as [14]

$-\sum_{i, j, k, l, \alpha, \beta} h_{i j}^{\alpha} h_{i j}^{\beta} h_{k l}^{\alpha} h_{k l}^{\beta}-\sum_{i, j, \alpha, \beta}\left(\sum_{k}\left(h_{i k}^{\alpha} h_{k j}^{\beta}-h_{j k}^{\alpha} h_{k i}^{\beta}\right)\right)^{2} \geq-b(p)|h|^{4}$,

with $b(1)=1$ and $b(p)=\frac{3}{2}$ if $p>1$. We need the following estimate:
LEMMA 2.1. [19] Let $M$ be an n-dimensional immersed submanifold with parallel mean curvature in the space form $M^{n+p}(k)$. Then

$$
|\nabla h|^{2}-|\nabla| h||^{2} \geq \frac{2}{n p}|\nabla| h||^{2}
$$

We also need the following Hoffman-Spruck Sobolev inequality.

LEMMA 2.2. [12] Let $M$ be an n-dimensional complete submanifold in a Hadamard manifold and let $\psi \in C_{0}^{1}(M)$. Then

$$
\left(\int_{M} \psi^{\frac{n}{n-1}} d v\right)^{\frac{n-1}{n}} \leq c(n) \int_{M}(|\nabla \psi|+n|H| \psi) d v
$$

where $c(n)=2^{n}(1+n)^{1+\frac{1}{n}}(n-1)^{-1} \omega_{n}^{-\frac{1}{n}}$ and $\omega_{n}$ is the volume of the unit ball in $\mathbb{R}^{n}$.

From Lemma 2.2, we have the following estimate.

LeMma 2.3. [11] For $n \geq 3$, let $M$ be an $n$-dimensional complete minimal submanifold in $\mathbb{S}^{n+p}$ and let $\psi \in C_{0}^{1}(M)$. Then

$$
\left(\int_{M} \psi^{\frac{2 n}{n-2}} d v\right)^{\frac{n-2}{n}} \leq 2\left(n^{2}+7\right) c^{2}(n) \int_{M}\left(|\nabla \psi|^{2}+|\psi|^{2}\right) d v .
$$

\section{PROOFS OF THE MAIN THEOREMS}

Proof of Theorem 1.3: Noting that

$$
\frac{1}{2} \Delta|h|^{2}=|\nabla| h||^{2}+|h| \Delta|h|
$$

it follows from (2.1) and (2.2) that

$$
|\nabla| h||^{2}+|h| \Delta|h| \geq|\nabla h|^{2}+n|h|^{2}-b(p)|h|^{4} .
$$

From Lemma 2.1, we have

$$
|h| \Delta|h| \geq \frac{2}{n p}|\nabla| h||^{2}+n|h|^{2}-b(p)|h|^{4} .
$$

Given $\eta \in C_{0}^{\infty}(M)$, multiplying (3.1) by $\eta^{2}$ and integrating over $M$ gives

$$
\begin{aligned}
\int_{M} \eta^{2}|h| \Delta|h| d v & +\int_{M} b(p) \eta^{2}|h|^{4} d v \geq \frac{2}{n p} \int_{M}|\nabla| h||^{2} \eta^{2} d v \\
& +n \int_{M}|h|^{2} \eta^{2} d v
\end{aligned}
$$

which implies

$$
\begin{aligned}
& -\int_{M} 2 \eta|h|\langle\nabla|h|, \nabla \eta\rangle d v+\int_{M} b(p) \eta^{2}|h|^{4} d v \\
& \geq\left(1+\frac{2}{n p}\right) \int_{M}|\nabla| h||^{2} \eta^{2} d v+n \int_{M}|h|^{2} \eta^{2} d v
\end{aligned}
$$


Further, applying Hölder's inequality and taking $\psi=|h| \eta$ in Lemma 2.3, one verifies that

$$
\begin{aligned}
\int_{M} \eta^{2}|h|^{4} d v & \leq\left(\int_{M}|h|^{n} d v\right)^{\frac{2}{n}}\left(\int_{M}(\eta|h|)^{\frac{2 n}{n-2}} d v\right)^{\frac{n-2}{n}} \\
& \leq 2\left(n^{2}+7\right) c^{2}(n)\left(\int_{M}|h|^{n} d v\right)^{\frac{2}{n}} \int_{M}\left(|\nabla(\eta|h|)|^{2}\right. \\
& \left.+|h|^{2} \eta^{2}\right) d v
\end{aligned}
$$

Setting

$$
l=2 b(p)\left(n^{2}+7\right) c^{2}(n)\left(\int_{M}|h|^{n} d v\right)^{\frac{2}{n}},
$$

from (3.3) and (3.5) we may estimate

$$
\begin{aligned}
& l \int_{M}|\nabla \eta|^{2}|h|^{2} d v+(l-1) \int_{M} 2 \eta|h|\langle\nabla|h|, \nabla \eta\rangle d v \\
& \quad \geq\left(1+\frac{2}{n p}-l\right) \int_{M}|\nabla| h||^{2} \eta^{2} d v+(n-l) \int_{M}|h|^{2} \eta^{2} d v
\end{aligned}
$$

By assumption,

$$
\left(\int_{M}|h|^{n} d v\right)^{\frac{1}{n}}<c^{-1}(n)\left(n^{2}+7\right)^{-\frac{1}{2}} \sqrt{(2 b(p))^{-1}+(n p b(p))^{-1}},
$$

and it is easy to see that

$$
1+\frac{2}{n p}-l>0
$$

Therefore, we can find a $\theta>0$ such that

$$
1+\frac{2}{n p}-l \geq \theta
$$

On the other hand, for any $\varepsilon>0$ we have

$$
\begin{aligned}
(l-1) \int_{M} 2 \eta|h|\langle\nabla|h|, \nabla \eta\rangle & d v \leq|l-1| \varepsilon \int_{M} \eta^{2}|\nabla| h||^{2} d v \\
& +|l-1| \varepsilon^{-1} \int_{M}|\nabla \eta|^{2}|h|^{2} d v
\end{aligned}
$$

Thus, when $|l-1| \varepsilon \leq \frac{\theta}{2}$, we obtain

$$
\begin{aligned}
\left(l+|l-1| \varepsilon^{-1}\right) \int_{M}|\nabla \eta|^{2}|h|^{2} d v & \geq \frac{\theta}{2} \int_{M}|\nabla| h||^{2} \eta^{2} d v \\
& +(n-l) \int_{M}|h|^{2} \eta^{2} d v
\end{aligned}
$$

Fix a point $x_{0} \in M$ and choose $\eta \in C_{0}^{\infty}(M)$ as

$$
\eta= \begin{cases}1 & \text { on } B_{x_{0}}(R), \\ 0 & \text { on } M \backslash B_{x_{0}}(2 R), \\ |\nabla \eta| \leq \frac{1}{R} & \text { on } B_{x_{0}}(2 R) \backslash B_{x_{0}}(R),\end{cases}
$$

with $0 \leq \eta \leq 1$, where $B_{x_{0}}(R)$ denotes the geodesic ball of radius $R$ centered at $x_{0} \in M$. Substituting the above $\eta$ into (3.7) and letting $R \rightarrow \infty$, we deduce that

$$
\int_{M}|\nabla| h||^{2} d v \leq 0, \quad \int_{M}|h|^{2} d v \leq 0 .
$$

Hence $|h|^{2}=0$, that is, $M^{n}$ is totally geodesic.

Proof of Theorem 1.4: Direct computation yields

$$
\Delta|h|^{\delta}=\delta(\delta-1)|h|^{\delta-2}|\nabla| h||^{2}+\delta|h|^{\delta-1} \Delta|h| .
$$

Multiplying (3.9) by $|h|^{\delta}$ and using (3.1), we infer that

$$
\begin{aligned}
|h|^{\delta} \Delta|h|^{\delta} & =\left.\left.\frac{\delta-1}{\delta}|\nabla| h\right|^{\delta}\right|^{2}+\delta|h|^{2 \delta-2}|h| \Delta|h| \\
& \geq\left.\left.\frac{\delta-1}{\delta}|\nabla| h\right|^{\delta}\right|^{2}+\frac{2 \delta}{n p}|\nabla| h||^{2}|h|^{2 \delta-2}+n \delta|h|^{2 \delta} \\
& -\delta b(p)|h|^{2 \delta+2} \\
& =\left.\left.\left(\frac{\delta-1}{\delta}+\frac{2}{n p \delta}\right)|\nabla| h\right|^{\delta}\right|^{2}+\left(n \delta-b(p) \delta|h|^{2}\right)|h|^{2 \delta} .
\end{aligned}
$$

Let $\eta \in C_{0}^{\infty}(M)$. Multiplying (3.10) by $\eta^{2}$ and integrating over $M$ yields

$$
\begin{aligned}
\int_{M} \eta^{2}|h|^{\delta} \Delta|h|^{\delta} d v \geq & \left.\left.\left(\frac{\delta-1}{\delta}+\frac{2}{n p \delta}\right) \int_{M} \eta^{2}|\nabla| h\right|^{\delta}\right|^{2} d v \\
+ & n \delta \int_{M} \eta^{2}|h|^{2 \delta} d v \\
& -b(p) \delta \int_{M} \eta^{2}|h|^{2 \delta+2} d v
\end{aligned}
$$

It follows from the divergence theorem and (3.11) that

$$
\begin{aligned}
& b(p) \delta \int_{M} \eta^{2}|h|^{2 \delta+2} d v-\int_{M} 2 \eta|h|^{\delta}\left\langle\nabla \eta, \nabla|h|^{\delta}\right\rangle d v \\
& \quad \geq\left.\left.\left(2+\frac{2-n p}{n p \delta}\right) \int_{M} \eta^{2}|\nabla| h\right|^{\delta}\right|^{2} d v+n \delta \int_{M} \eta^{2}|h|^{2 \delta} d v .
\end{aligned}
$$

Applying Hölder's inequality and taking $\psi=|h|^{\delta} \eta$ in Lemma 2.3, we have

$$
\begin{aligned}
\int_{M} \eta^{2}|h|^{2 \delta+2} d v & \leq\left(\int_{M}|h|^{n} d v\right)^{\frac{2}{n}}\left(\int_{M}\left(\eta|h|^{\delta}\right)^{\frac{2 n}{n-2}} d v\right)^{\frac{n-2}{n}} \\
& \leq 2 n^{2} c^{2}(n)\left(\int_{M}|h|^{n} d v\right)^{\frac{2}{n}} \int_{M}\left(\left|\nabla\left(\eta|h|^{\delta}\right)\right|^{2}\right. \\
& \left.+|h|^{2 \delta} \eta^{2}\right) d v
\end{aligned}
$$

Substituting (3.13) into (3.12) yields

$$
\delta l \int_{M}|h|^{2 \delta}|\nabla \eta|^{2} d v+(\delta l-1) \int_{M} 2 \eta|h|^{\delta}\left\langle\nabla \eta, \nabla|h|^{\delta}\right\rangle d v
$$




$$
\begin{aligned}
& \geq\left.\left.\left(2+\frac{2-n p}{n p \delta}-l \delta\right) \int_{M} \eta^{2}|\nabla| h\right|^{\delta}\right|^{2} d v \\
& +(n \delta-l \delta) \int_{M} \eta^{2}|h|^{2 \delta} d v
\end{aligned}
$$

where $l=2 b(p)\left(n^{2}+7\right) c^{2}(n)\left(\int_{M}|h|^{n}\right)^{\frac{2}{n}}$. Further, using the Cauchy-Schwarz inequality, for each $\varepsilon>0$ we obtain

$$
\begin{aligned}
& (\delta l-1) \int_{M} 2 \eta|h|^{\delta}\left\langle\nabla \eta, \nabla|h|^{\delta}\right\rangle d v \leq\left.\left.|\delta l-1| \varepsilon \int_{M} \eta^{2}|\nabla| h\right|^{\delta}\right|^{2} d v \\
& +|\delta l-1| \varepsilon^{-1} \int_{M}|h|^{2 \delta}|\nabla \eta|^{2} d v
\end{aligned}
$$

Therefore

$$
\begin{aligned}
(\delta l & \left.+|\delta l-1| \varepsilon^{-1}\right) \int_{M}|h|^{2 \delta}|\nabla \eta|^{2} d v \\
& \geq\left.\left.\left(2+\frac{2-n p}{n p \delta}-l \delta-|\delta l-1| \varepsilon\right) \int_{M} \eta^{2}|\nabla| h\right|^{\delta}\right|^{2} d v \\
& +(n \delta-l \delta) \int_{M} \eta^{2}|h|^{2 \delta} d v
\end{aligned}
$$

By the assumption in the theorem that

$$
(n c(n))^{-1} \sqrt{\frac{2(n-1)}{\left(n^{2}+7\right) b(p)}+\frac{4}{n\left(n^{2}+7\right) p b(p)}}>\left(\int_{M}|h|^{n} d v\right)^{\frac{1}{n}},
$$

we have

$$
2+\frac{2-n p}{n p \delta}-l \delta>0 .
$$

Choosing $\varepsilon$ sufficiently small, we can get

$$
2+\frac{2-n p}{n p \delta}-l \delta-|\delta l-1| \varepsilon>0 .
$$

Defining the cut-off function as in (3.8) and taking $\delta=\frac{n}{2}$ in (3.16), we obtain

$$
\begin{aligned}
& \left(\frac{n}{2} l+\left|\frac{n}{2} l-1\right| \varepsilon^{-1}\right) \frac{\int_{M}|h|^{n} d v}{R^{2}} \\
& \geq\left(\frac{n}{2} l+\left|\frac{n}{2} l-1\right| \varepsilon^{-1}\right) \frac{\int_{B_{x_{0}}(2 R)}|h|^{n} d v}{R^{2}} \\
& \geq\left.\left.\left(2+\frac{4-2 n p}{n^{2} p}-\frac{n}{2} l-\left|\frac{n}{2} l-1\right| \varepsilon\right) \int_{B_{x_{0}}(R)}|\nabla| h\right|^{\frac{n}{2}}\right|^{2} d v \\
& +\left(\frac{1}{2} n^{2}-\frac{n}{2} l\right) \int_{B_{x_{0}}(R)}|h|^{n} d v .
\end{aligned}
$$

Since

$$
\left(\int_{M}|h|^{n} d v\right)^{\frac{1}{n}}<\widetilde{C}(n, p)
$$

upon taking $R \rightarrow \infty$ we have

$$
\frac{\int_{M}|h|^{n} d v}{R^{2}} \rightarrow 0
$$

This and (3.17) imply $\nabla|h|=0$ and $|h|=0$, that is, $M^{n}$ is totally geodesic.
Proof of Theorem 1.6: Since $M$ is a stable minimal hypersurface in the unit sphere $\mathbb{S}^{n+1},(1.2)$ holds on $M$. Let $\eta \in C_{0}^{\infty}(M)$. Replacing $f$ by $\eta|h|^{\delta}$ in (1.2) and taking $\overline{\operatorname{Ric}}(v, v)=n$ give

$$
\int_{M}\left|\nabla\left(\eta|h|^{\delta}\right)\right|^{2} d v \geq \int_{M} \eta^{2}|h|^{2 \delta+2} d v+n \int_{M} \eta^{2}|h|^{2 \delta} d v,
$$

that is,

$$
\begin{gathered}
\int_{M}|\nabla \eta|^{2}|h|^{2 \delta} d v+\left.\left.\int_{M} \eta^{2}|\nabla| h\right|^{\delta}\right|^{2} d v \\
+2 \int_{M} \eta|h|^{\delta}\left\langle\nabla \eta, \nabla|h|^{\delta}\right\rangle d v-n \int_{M} \eta^{2}|h|^{2 \delta} d v \\
\geq \int_{M} \eta^{2}|h|^{2 \delta+2} d v .
\end{gathered}
$$

Substituting (3.18) into (3.12) and noting that $b(1)=1$, we obtain

$$
\begin{aligned}
& \delta \int_{M}|\nabla \eta|^{2}|h|^{2 \delta} d v+2(\delta-1) \int_{M} \eta|h|^{\delta}\left\langle\nabla \eta, \nabla|h|^{\delta}\right\rangle d v \\
& \quad \geq\left.\left.\left(2+\frac{2-n}{n \delta}-\delta\right) \int_{M} \eta^{2}|\nabla| h\right|^{\delta}\right|^{2} d v+2 n \delta \int_{M} \eta^{2}|h|^{2 \delta} d v
\end{aligned}
$$

Using $1-\sqrt{\frac{2}{n}}<\delta<1+\sqrt{\frac{2}{n}}$, we see that

$$
2+\frac{2-n}{n \delta}-\delta>0
$$

Further, for any $\varepsilon>0$, it follows from the Cauchy-Schwarz inequality that

$$
\begin{aligned}
\varepsilon \mid \delta & -\left.1\left|\int_{M} \eta^{2}\right| \nabla|h|^{\delta}\right|^{2} d v+\varepsilon^{-1}|\delta-1| \int_{M}|\nabla \eta|^{2}|h|^{2 \delta} d v \\
& \geq 2(\delta-1) \int_{M} \eta|h|^{\delta}\left\langle\nabla \eta, \nabla|h|^{\delta}\right\rangle d v .
\end{aligned}
$$

Combining (3.20) and (3.19) gives

$$
\begin{aligned}
(\delta & \left.+\varepsilon^{-1}|\delta-1|\right) \int_{M}|\nabla \eta|^{2}|h|^{2 \delta} d v \\
& \geq\left.\left.\left(2+\frac{2-n}{n \delta}-\delta-\varepsilon|\delta-1|\right) \int_{M} \eta^{2}|\nabla| h\right|^{\delta}\right|^{2} d v \\
& +2 n \delta \int_{M} \eta^{2}|h|^{2 \delta} d v
\end{aligned}
$$

Choosing $\varepsilon$ sufficiently small, we can obtain

$$
2+\frac{2-n}{n \delta}-\delta-\varepsilon|\delta-1|>0 .
$$

Furthermore, defining the cut-off function as in (3.8) and using the assumption (1.3) yield $\nabla|h|=0$ and $|h|=0$, that is, $M^{n}$ is totally geodesic. 


\section{CONCLUSION}

In this paper, by using Simons' formula, a Sobolev-type inequality as in Chen and $\mathrm{Xu}$ [4], and the technique of do Carmo and Peng, we obtain rigidity theorems for minimal submanifolds in $\mathbb{S}^{n+p}$. Compared with Theorem 1.1, Theorem 1.4 removes the condition on the growth of the norm of the second fundamental form. Moreover, our results require only $n \geq 3$, whereas Theorems 1.1 and 1.2 require $n \geq 5$ and $n \geq 4$, respectively. Whether the pinching constant for the total curvature in Theorem 1.4 is optimal remains an open question and is a topic of future research.

\section{DATA AVAILABILITY STATEMENT}

The raw data supporting the conclusions of this article will be made available by the authors, without undue reservation.

\section{REFERENCES}

1. Almgren F. Some interior regularity theorems for minimal surfaces and an extension of Bernstein's theorem. Ann Math. (1966) 84:277-92. doi: $10.2307 / 1970520$

2. Bombieri E, De Giorgi E, Giusti E. Minimal cones and the Bernstein problem. Invent Math. (1969) 7:243-68. doi: 10.1007/BF01404309

3. Chern S., do Carmo M., Kobayashi S. Minimal submanifolds of a sphere with second fundamental form of constant length. In: Tenenblat K, editor. Manfredo P. do Carmo - Selected Papers. Berlin; Heidelberg: Springer (1970). p. 59-75. doi: 10.1007/978-3-642-25588-5_5

4. Chen Q, Xu S. Rigidity of compact minimal submanifolds in a unit sphere. Geome Dedicata. (1993) 45:83-8. doi: 10.1007/BF01667404

5. Deshmukh S. Rigidity of compact minimal submanifolds in a sphere. Pacific J Math. (2000) 193:31-44. doi: 10.2140/pjm.2000.193.31

6. do Carmo M, Peng C. Stable complete minimal hypersurfaces. Proc Beijing Symp Diff Equat Diff Geometry. (1980) 3:1349-58.

7. do Carmo M, Zhou D. Bernstein-type theorems in hypersurfaces with constant mean curvature. An Acad Bras Ciênc. (2000) 72:301-10. doi: 10.1590/S0001-37652000000300003

8. de Oliveira H, Xia C. Rigidity of complete minimal submanifolds in a hyperbolic space. Manuscripta Math. (2019) 158:21-30. doi: 10.1007/s00229-018-1013-4

9. Fleming W. On the oriented Plateau problem. Rend Circ Mat Palermo. (1962) 11:69-90. doi: 10.1007/BF02849427

10. Fischer colbrie D, Schoen R. The structure of complete stable minimal surfaces in 3-manifolds of non-negative scalar curvature. Commun Pure Appl Math. (1980) 33:199-211. doi: 10.1002/cpa.3160330206

11. Fu H, Xu H. Total curvature and $\mathrm{L}^{2}$ harmonic 1-forms on complete submanifolds in space forms. Geom Dedicata. (2010) 144:129-40. doi: 10.1007/s10711-009-9392-Z

\section{AUTHOR CONTRIBUTIONS}

The author confirms being the sole contributor of this work and has approved it for publication.

\section{FUNDING}

This work was supported by the Natural Science Foundation of Anhui Province Education Department (grant nos. KJ2017A341 and KJ2018A0330), the Talent Project of Fuyang Normal University (grant no. RCXM201714), and the Key Laboratory of Applied Mathematics of Fujian Province University (Putian University) (grant no. SX201805).

\section{ACKNOWLEDGMENTS}

The author expresses sincere gratitude to the reviewers and the editors for their careful reading of the manuscript and constructive recommendations.

12. Hoffman D, Spruck J. Sobolev and isoperimetric inequalities for riemannian submanifolds. Commun Pure Appl Math. (1974) 27:715-27. doi: 10.1002/cpa.3160270601

13. Lawson B. The equivariant Plateau problem and interior regularity. Trans Am Math Soc. (1972) 173:231-50. doi: 10.2307/1996271

14. Li A, Li J. An intrinsic rigidity theorem for minimal submanifolds in a sphere. Arch Math. (1992) 58:582-94. doi: 10.1007/BF01193528

15. Ni L. Gap theorems for minimal submanifolds in $\mathbb{R}^{n+1}$. Commun Anal Geom. (2001) 9:641-56. doi: 10.4310/CAG.2001.v9.n3.a2

16. Neto N, Wang Q. Some Bernstein-type rigidity theorems. J Math Anal Appl. (2012) 389:694-700. doi: 10.1016/j.jmaa.2011.12.013

17. Simons J. Minimal varieties in Riemannian manifolds. Ann Math. (1968) 88:62-105. doi: 10.2307/1970556

18. Shen $Y$, Zhu X. On stable complete minimal hypersurfaces in $\mathbb{R}^{n+1}$. Am J Math. (1998) 120:103-16. doi: 10.1353/ajm.1998.0005

19. Xin Y, Yang L. Curvature estimates for minimal submanifolds of higher codimension. Chinese Ann Math. (2009) 30:379-96. doi: 10.1007/s11401-008-0438-6

20. Xia C, Wang Q. Gap theorems for minimal submanifolds of a hyperbolic space. J Math Anal Appl. (2016) 436:983-9. doi: 10.1016/j.jmaa.2015.12.050

Conflict of Interest: The author declares that the research was conducted in the absence of any commercial or financial relationships that could be construed as a potential conflict of interest.

Copyright (c) 2020 Zhou. This is an open-access article distributed under the terms of the Creative Commons Attribution License (CC BY). The use, distribution or reproduction in other forums is permitted, provided the original author(s) and the copyright owner(s) are credited and that the original publication in this journal is cited, in accordance with accepted academic practice. No use, distribution or reproduction is permitted which does not comply with these terms. 